\title{
Composite spectrum hot subdwarf binaries
}

\author{
P. Németh ${ }^{1,2}$ \\ 1 Astronomical Institute of the Czech Academy of Sciences \\ 25165 Ondřejov, Czech Republic \\ 2 Astroserver.org, 8533 Malomsok, Hungary \\ (E-mail: peter.nemeth@astroserver.org)
}

Received: November 2, 2019; Accepted: January 27, 2020

\begin{abstract}
The formation of hot subdwarf stars (sdB) is closely related to binary evolution on the red giant branch. Observations show that hot subdwarfs are either in close binaries with low mass K-M type or white dwarf companions, or in wide binaries with more massive F-G type companions. A small fraction is most strikingly single. In close binaries the radiative interactions give rise to the reflection effect and produces a variable composite spectrum. More massive companions bear similar optical luminosities as sdB stars and therefore double-lined spectra are observed. To investigate such systems in detail and precision spectral decomposition/disentangling is needed. I describe our wavelength space disentangling method and bring examples why such an approach to composite spectra offers a potential to find the history of hot subdwarfs.
\end{abstract}

Key words: stars - hot subdwarfs - spectroscopy - composite spectra

\section{Introduction}

Hot subdwarf (sdB) stars are $0.5 \mathrm{M}_{\odot}$ core helium burning stars with very thin hydrogen envelopes. The mass loss required to form such stars is still poorly understood. The fact that the envelope loss happens by the time the core ignites helium makes it likely that binarity and enhanced wind mass loss act together on the red giant branch. Passing through the core helium flash, these low mass stars settle on the blue extension of the horizontal branch and burn helium at a constant luminosity for $\sim 100$ Myrs. Following this stage these stars evolve to white dwarfs without ever ascending the giant branch again (Heber, 2016).

Hot subdwarfs are interesting objects that show a multitude of atmospheric processes, which make them complex, yet unique laboratories to get insights into intermediate age stars. A significant fraction of hot subdwarfs show pulsations, signatures of atomic diffusion in the atmosphere along with diverse binary properties. Hence, their analysis requires similarly complex approaches that, in turn, offers a chance to connect those fields, and open new windows in exploration.

A significant fraction (near 20\%) of hot subdwarfs are identified in doublelined composite spectrum systems, while in numerous close binaries spectral contamination is visible. All binaries with less than 0.5 day orbital period and 
low mass main sequence companions are expected to show reflection effect. All such systems require appropriate decomposition or disentangling techniques.

\section{Hot subdwarf binary populations}

Since the first spectroscopic and radial velocity surveys it is known that the binary fraction of hot subdwarfs is high, between 43 and $67 \%$. The large scatter is due to different methods and selection effects. In fact, the high fraction was expected, as population synthesis by Han et al. (2002) predicted an even higher fraction, near $80 \%$. The remaining $20 \%$ forms by double white dwarf mergers.

In a recent radial velocity study Kawka et al. (2015) found that $43 \%$ of the subdwarf population is indeed in binaries. Projecting onto the whole hot subdwarf population, $13-17 \%$ have white dwarf companions and 26-30\% have low mass main sequence companions or near the substellar limit. About 20\% has more massive main sequence companions (Németh et al., 2012).

Binaries with main sequence companions separate into two very diverse subgroups: (1) Systems that form a subdwarf through common-envelope evolution have typically a low mass companion and due to the spiral-in process the orbital period is less than 30 days. At the shortest periods the small separation gives rise to a reflection effect in the light curve and this reflected (in fact it is reprocessed) light makes such systems composite spectrum binaries. (2) In contrast, binaries that form subdwarfs through Roche-lobe overflow have more massive F-G-K type main sequence companions and much longer orbits, in the range of 400-1500 days. These systems show remarkable double-lined composite spectra as the optical luminosities of the members are similar.

Single hot subdwarfs, as well as single-lined binaries cannot give the necessary insights into their formation history or progenitor systems because the atmospheric investigations are limited to one component. The key to understand hot subdwarfs, therefore, is thought to be in composite spectrum populations. In a variety of long-period double-lined binaries and reflection effect short-period binaries, a careful analysis is able to reveal radial velocities, stellar masses, ages and chemical compositions. However, such tasks require specially designed, delicate methods.

\section{Modeling composite spectra}

\subsection{Fit or decomposition}

One can talk about spectral fitting as long as a single model reproduces all observed features of an object. With composite spectra this is not the case. In some situations one can apply data reduction techniques, remove components, or part of the data that is not reproduced by the model to investigate the object of interest. However, at least a decomposition approach with a combination of multiple models is needed to reproduce an observation. Such as the combination 
of two (or more) models for double-lined binaries and reflection effect systems. Although the combination of the models is a tricky and cumbersome part of the process, it provides invaluable information on the components and of the system as a whole (Østensen, 2012).

A simple approach to spectral decomposition is to reproduce the spectral energy distribution. Using broad band photometry and color indices the components can be characterized this way easily and applied in large surveys. Unfortunately, this method lacks precision. As no radial velocities, abundances or precise atmospheric parameters can be derived, this method is suitable only for coarse population studies, and even then it needs spectroscopic confirmation.

\subsection{Disentangling wide binaries}

There are two major sets of dependent parameters when one is dealing with composite spectra: the radial velocities and the spectral types of the binary members. The radial velocities can be measured by cross-correlation techniques, which provide the orbital properties. The spectral appearance can be reproduced by keeping radial velocities fixed and decomposing the spectrum into its components either by successive subtractions or by co-adding models. To generalize these steps to any kind of composite binary spectrum, one needs disentangling, in which both the velocities and the spectral types are determined simultaneously. Disentangling is a complex task which has two major approaches: Fourier Space (KOREL, Hadrava 2009) and the direct Wavelength Space Disentangling (Simon \& Sturm, 1994). Both methods seek for the same set of parameters and the steps are analogous within the two frameworks. However, both have different benefits and limitations. The Fourier method is based on the recognition of regularities over the orbital period in the Fourier transformed spectra, while wavelength space disentangling applies a linear combination of synthetic spectra to reproduce the observed composite spectrum at any instance.

The Fourier method provides a clear mathematical formalism that allows an easier reduction and generalization for multiple systems. However, it is done at the cost of a fair observational sampling of the orbital period and assumes clean spectral features of the members. In contrast, the direct decomposition requires only one spectrum and can overcome heavily blended spectral features.

Both approaches have extensions to cope with higher order effects, such as broken symmetries of the spectroscopic line profiles. One typical situation for these are partial eclipses in binaries, where the radial velocities, limb-darkening and dilution factors change during an eclipse and the radial velocity does not reflect the center of mass velocity. Similar line profile variations may be present due to external irradiation, pulsations, spots, ellipticity, mass-loss, etc.

It is obvious that the direct wavelength space disentangling is more suitable for long period hot subdwarf binaries with cool main sequence companions. Due to the long period there is usually an insufficient orbital coverage for the Fourier approach and the broad Balmer and He lines of the hot subdwarf are heavily 
blended with the Balmer and the numerous metal lines of the companion, as in Fig. 1.

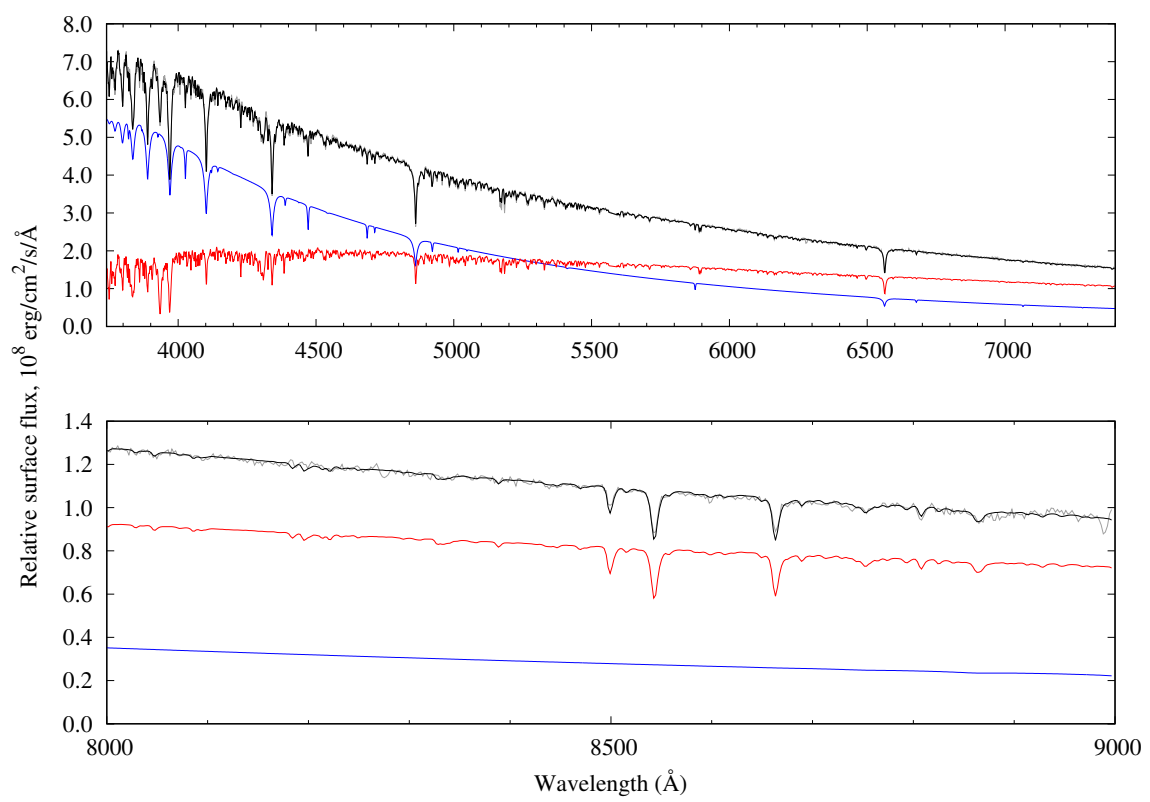

Figure 1. TLUSTY/XTGRID binary fit for the LAMOST DR1 composite spectrum of BD-7 5977 (grey line). The black model shows how the sum of an sdB (blue) and a G0V type model (red) fits the observation across the entire optical range. Further examples can be found at https://astroserver.org/KW32YZ/.

\subsection{External irradiation in close binaries}

Another very important advantage of the direct wavelength space decomposition is the easier implementation of modeling orbital spectral variations. These variations occur in close binaries (also in planet+host star systems) where a hotter component heats up the substellar point of its cooler companion. This phenomena cause not only the reflection effect in the light curve, but introduce characteristic orbital phase dependent emission components as in Fig. 2, and spectral variations in the composite spectrum. Once the spectral variations are reproduced by models, all such binaries can be turned into double-lined systems and mass estimates can be made.

Unlike at long-period composite spectrum binaries, where the linear combination of the mean fluxes can fully describe the orbital spectral variations, in reflection effect systems one must apply a surface integration of the specific intensities to recover the composite spectrum. This is a lot more demanding quantitative task. However, future instruments will be more capable to deliver 
observational data to such investigations and extend the number of feasible hot subdwarf binaries from the currently available few to over a 100.

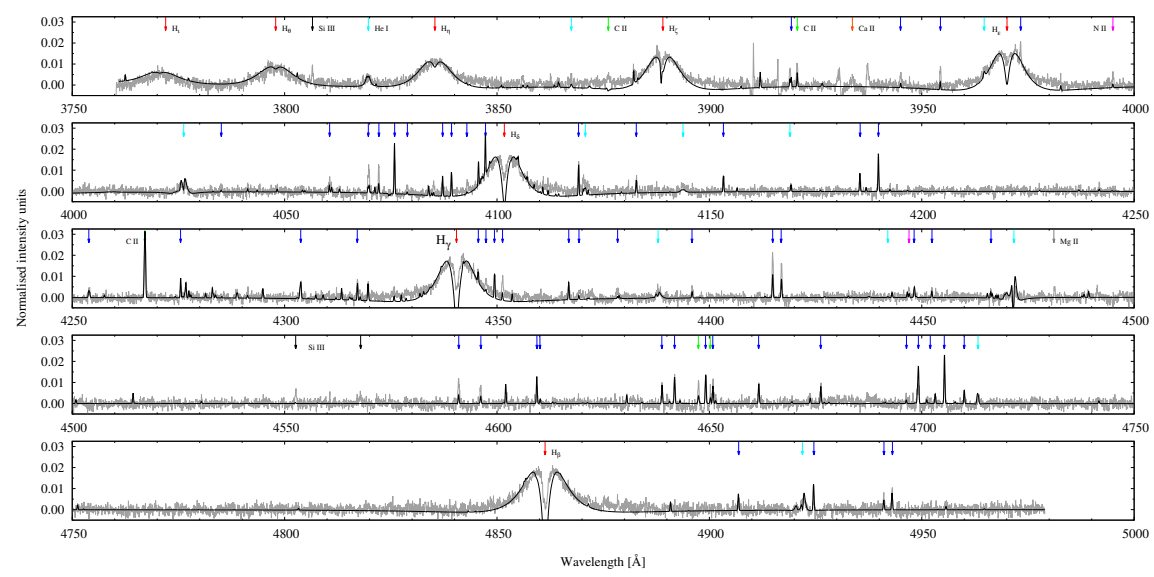

Figure 2. TLuSTY / XTGRID fit for the irradiated hemisphere of the cool companion in AA Doradus (Vučković et al., 2016). An animated version showing the spectral variations over the orbit is available online at https://youtu.be/QMsXSnw_zm8.

\subsection{Composite spectroscopy with XTGRID}

XTGRID (Németh et al., 2012) is a spectral analysis procedure that wraps around the non-Local Thermodynamic Equilibrium model atmosphere and synthetic spectrum codes Tlusty/Synspec (Hubeny \& Lanz 2017a,b,c). In addition to TLUSTY models the procedure can include further components to build composite spectra and fit the observations of binary and multiple systems. The fitting method is based on an iterative chi-square minimization using a downhill simplex method. The main focus of the procedure is to fit hot star binaries or hot stars with cool companions. The companion spectra are calculated in parallel threads using TLUSTY, or extracted from pre-calculated spectral grids, such as the PHOENIX (Husser et al., 2013) or BOSZ (Bohlin et al., 2017) libraries. XTGRID has been employed to solve subdwarf binary orbits for 28 systems with UVES spectroscopy (Vos et al., 2018) and showed consistent results with the independent GSSP code (Tkachenko, 2015) for the cool companions. These analyses found a positive correlation between orbital period and eccentricity and an anti-correlation between orbital period and mass ratio (Vos et al., 2019). In some systems the companion shows chromospheric activity based on the near infrared calcium triplet.

TLUSTY is a sophisticated code that can model external irradiation in close binaries and the spectra of radiatively heated companions. This allows one to calculate specific intensities and integrate the emergent spectra from the visible surface elements of the companion, as demonstrated by Vučković et al. (2016). 


\section{Conclusions}

Spectral disentangling seems to be the key, and the way to go, to understand hot subdwarf formation and evolution. It is absolutely necessary for any precision analysis of long-period double-lined subdwarf binaries with main-sequence companions. Many such systems are simply neglected in surveys because of their complexity, or modelled as single stars leading to large systematic discrepancies. The situation is more severe for close binaries where a composite spectrum may remain unnoticed. If reflection effect is observed in the light curve one can be assured that the companion contributes to the spectrum and this may invalidate the surface parameters derived with single star models. Even though a careful analysis and windowing can remedy the situation, with a simultaneous analysis of the components one can achieve more comprehensive results of the systems. Such a procedure is also available within the Sandbox services of Astroserver.org at https://xtgrid.astroserver.org/sandbox/.

Acknowledgements. P.N. acknowledges support from the Grant Agency of the Czech Republic (GAČR 18-20083S).

\section{References}

Bohlin, R. C., Mészáros, S., Fleming, S. W., et al. 2017, Astron. J., 153, 234

Hadrava, P. 2009, arXiv e-prints, arXiv:0909.0172

Heber, U. 2016, Publ. Astron. Soc. Pac., 128, 082001

Han, Z., Podsiadlowski, P., Maxted, P. F. L., et al. 2002, Mon. Not. R. Astron. Soc., 336,449

Hubeny, I., \& Lanz, T. 2017a, arXiv e-prints, arXiv:1706.01859

Hubeny, I., \& Lanz, T. 2017b, arXiv e-prints, arXiv:1706.01935

Hubeny, I., \& Lanz, T. 2017c, arXiv e-prints, arXiv:1706.01937

Husser, T.-O., Wende-von Berg, S., Dreizler, S., et al. 2013, Astron. Astrophys., 553, A6

Kawka, A., Vennes, S., O’Toole, S., et al. 2015, Mon. Not. R. Astron. Soc., 450, 3514

Németh, P., Kawka, A., \& Vennes, S. 2012, Mon. Not. R. Astron. Soc., 427, 2180

Østensen, R. H. 2012, Fifth Meeting on Hot Subdwarf Stars and Related Objects, 233

Simon, K. P., \& Sturm, E. 1994, Astron. Astrophys., 281, 286

Tkachenko, A. 2015, Astron. Astrophys., 581, A129

Vos, J., Németh, P., Vučković, M., et al. 2018, Mon. Not. R. Astron. Soc., 473, 693

Vos, J., Vučković, M., Chen, X., et al. 2019, Mon. Not. R. Astron. Soc., 482, 4592

Vučković, M., Østensen, R. H., Németh, et al. 2016, Astron. Astrophys., 586, A146 